\title{
A case of endometrial glassy cell carcinoma: a rare entity which necessitates of better understanding
}

\author{
Clelia Callegari ${ }^{1,2}$, Paola Algeri ${ }^{3}$, Antonella Buzzi ${ }^{4}$, Teresio Motta ${ }^{4}$, Cinzia Manfredini ${ }^{4}$, Sonia Maria Rota ${ }^{3}$ \\ ${ }^{1}$ Department of Obstetrics, Foundation MBBM, Monza, ${ }^{2}$ University of Milan-Bicocca School of Medicine and Surgery, Monza, ${ }^{3}$ Department of \\ Obstetrics and Gynecology, Bolognini Hospital, Seriate, ${ }^{4}$ Department of Pathology, Bolognini Hospital, Seriate, Italy
}

Endometrial glassy cell carcinoma (EGCC) is a rare neoplasm, accounting for $0.5 \%$ of the carcinomas in the endometrium, composed of cells with granular eosinophilic or amphophilic cytoplasm, giving it a ground glass appearance. Till date, only 14 cases of this carcinoma have been reported. In this report, we have described a case of EGCC to help define standard diagnostic criteria and better understand the course, ideal treatment, and accurate prognosis of this disease. We report a case of a 64-year-old woman diagnosed with EGCC after an abnormal pap smear. She underwent a hysteroscopy, which led to the histological diagnosis. Laparotomic total hysterectomy and bilateral salpingo-oophorectomy were performed with pelvic lymphadenectomy and peritoneal and omental biopsies. Final pathological examination confirmed the initial diagnosis. Pelvic nodes removed during surgery and peritoneal and omental biopsies were negative for tumor cells. Treatment was considered appropriate and the patient did not require additional therapies. She was subsequently assigned to clinical follow-up and is alive, with no evidence of the disease.

Keywords: Endometrial cancer; Glassy cell carcinoma; Uterine cancer

\section{Introduction}

Glassy cell carcinoma (GCC) is a rare cancer originating in only $1 \%$ of cases in the uterine cervix.

Cervical GCC, a highly aggressive tumor characterized by resistance to radiation therapy, presents an unfavorable prognosis [1-3]. This malignancy has been considered as an uncommon variant of poorly differentiated adenosquamous carcinoma [1]. The first case of this condition was described by Cherry and Glucksmann [1] in 1956.

Rare cases of GCC arising in the fallopian tubes and colon have also been described in the literature $[4,5]$.

Christopherson et al. [6] described the first case of endometrial GCC (EGCC) in 1982. EGCC is a very rare occurrence, and to the best of our knowledge, only 14 cases have been reported till date [6-13]. EGCC accounts for approximately $0.5 \%$ of all carcinomas in the endometrium [6] and is frequently considered as a variant of the adenosquamous endometrial carcinoma.

EGCC is a poorly differentiated neoplasm with little or no glandular or squamous differentiation. Microscopically the tumor cells have well-defined borders and a granular eosino- philic or amphophilic cytoplasm, imparting a ground glass appearance. The tumor cells are usually arranged in diffuse solid sheets and have large nuclei with prominent nucleoli [14]. The exact percentage of glassy cells for the diagnosis of GCC is not well established, and ranges from $30 \%$ to $100 \%$ [15].

The tumor could be positive for cytokeratin (CK) and carcinoembryonic antigen (CEA), even if available data are discordant in the description of the immunohistochemical characteristics of the neoplasm.

Received: 2019.01.31. Revised: 2019.04.28. Accepted: 2019.05.02. Corresponding author: Clelia Callegari

Department of Obstetrics, Foundation MBBM, Via Pergolesi 33, 20900 Monza, Italy

E-mail: clelia.callegari@gmail.com

https://orcid.org/0000-0002-9727-4096

Articles published in Obstet Gynecol Sci are open-access, distributed under the terms of the Creative Commons Attribution Non-Commercial License (http://creativecommons. org/licenses/by-nc/3.0/) which permits unrestricted non-commercial use, distribution, and reproduction in any medium, provided the original work is properly cited.

Copyright ( 2019 Korean Society of Obstetrics and Gynecology 


\section{Obstetrics \& Gynecology Science}

Clelia Callegari, et al. Endometrial glassy cell carcinoma

Given the rarity of EGCC, the challenges encountered in the diagnosis and the poor prognosis, we aimed to review the published literature on the condition. We have also reported a case of EGCC in a 64-year-old woman under regular follow-up and no signs of relapse of the disease, in order to contribute towards improvement of the clinical management of this tumor.

\section{Case report}

A 64-year-old nulliparous patient underwent routine gynecological examination at the Seriate Bolognini Hospital in March 2017.

The patient was asymptomatic and reported a noncontributory familial and personal history. The gynecological examination was normal, while a routine Pap smear demonstrated positive results for adenocarcinoma. Subsequently, the patient underwent colposcopy, which was negative for signs of cervical carcinoma. She also underwent hysteroscopy, which revealed a tender and frail endometrial neoplasm with heavy atypical vascularization. A biopsy of the lesion was obtained, which was positive for EGCC. The specimen was found to be CK20, CD10, CEA, WT1, P63, estrogen and progesterone receptor-negative, while it was positive for $A E 1 / A E 3, C K 7$, $\mathrm{P} 53$, and P16.

Preoperative radiological investigations included chest $X$ ray, which was normal and pelvic magnetic resonance imaging that revealed a solid tumor completely occupying the uterine cavity and invading more than $50 \%$ of the myometrium. No other signs of disease were revealed. Blood test was normal, with negative tumor markers.

The patient underwent laparotomy; at the opening of the abdominal wall, we detected heavy, dense parieto-omental and utero-sigmoid adhesions. Peritoneal washing was collected. Lysis of the adhesions was performed to achieve optimal surgical exposure. Macroscopically, the uterus and the adnexa appeared normal; endoperitoneal and retroperitoneal surgical evaluation were negative, with no signs of the tumor.

Total hysterectomy and bilateral salpingo-oophorectomy were performed with pelvic lymphadenectomy and peritoneal and omental biopsies.

Conclusive pathological examination confirmed the initial diagnosis of EGCC involving $2 \mathrm{~mm}$ of myometrium (on a to- tal thickness of $12 \mathrm{~mm}$ ), International Federation of Gynecology and Obstetrics stage IA, grade 3. There were no signs of lymphovascular space invasion or presence of the disease in the blind peritoneal biopsies and the 18 pelvic nodes excised at the time of surgery.

Final immunoreaction test was comparable to the one performed during the first biopsy; the tumor was characterized by large cells with well-defined borders, granular eosinophilic or amphophilic cytoplasm, large nuclei and numerous mitotic cells. Heavy inflammatory cell infiltration was observed. These features were reported throughout the neoplasm (Fig. 1).

In light of limited myometrial infiltration (under 50\%), absence of lymphovascular space invasion, and the controversial utility of chemo-radiotherapy, the patient was referred to our outpatient clinic after surgery for clinical follow-up, which, until now (24 months), has not shown any evidence of relapse.

We obtained a written consent from the patient to collect and use her medical data for publication while maintaining anonymity.

\section{Discussion}

GCC is a rare tumor, which usually originates from the cervix, but may occasionally arise in the fallopian tubes, endometrium, and colon $[4,5]$.

The endometrial GCC accounts $0.5 \%$ of all carcinomas of the endometrium and, due to his rarity, few information was reported in literature about its pathogenesis. Cherry and Glucksmann [1] described the histological characteristics

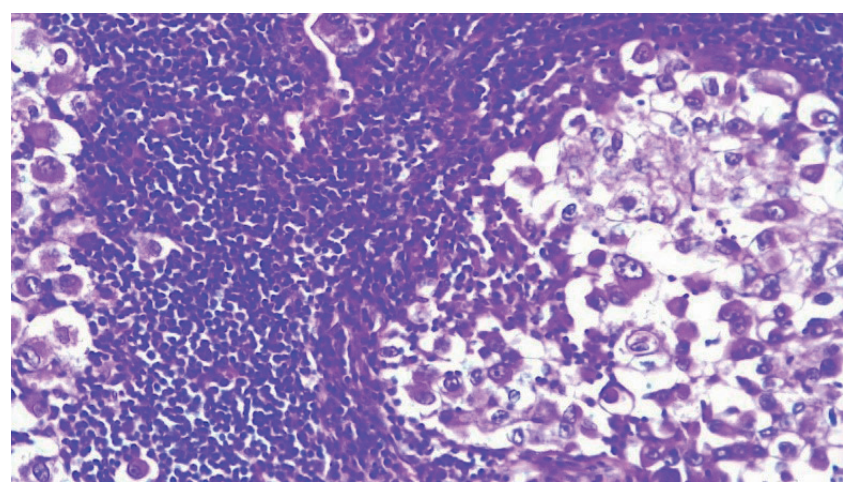

Fig. 1. Hematoxylin and eosin staining $(20 x)$ : Neoplasia characterized by ground glass cells with large nuclei associated with inflammatory cell infiltration. 


\title{
Obstetrics \& Gynecology Science
}

\author{
Vol. 62, No. 6, 2019
}

of the tumor as follows: moderate amount of cytoplasm, absence, or paucity of squamous or glandular differentiation, cell wall positivity for periodic acid Schiff and eosin, and large nuclei with prominent nucleoli. However, this pattern is not sufficient to trace the histogenesis of this uncommon tumor, which has traditionally been regarded, from the first description until today, as a poorly differentiated variant of adenosquamous carcinoma. Nevertheless, there is limited consensus regarding this hypothesis. Arends et al. [7] suggested that EGCC is not a true poorly differentiated tumor and do not consider the possibility that the condition is a variant of adenosquamous carcinoma. Furthermore, considering the absence of keratin and prekeratin and the presence of secretory component and lysozyme in the tumor cells, the group has supported an endocervical or isthmic origin of this neoplasia.

However, in the available literature, excluding the cases of advanced stages of the disease, EGCC were reported to be confined in the uterine corpus, suggesting that the hypothesis postulated by Arends et al. [7] is not accurate. Given the disagreement regarding the origin and histological diagnosis of this disease, systematic evaluation of the histological pattern is necessary to define strict and shared diagnostic criteria.
Considering the limited available information of this rare condition, we have summarized (Table 1) the reported cases and added the description of our patient.

The most frequent symptom at the time of admission was abnormal vaginal bleeding; and the age of the patients ranged from 52 to 96 years (mean, 66.2 years), in line with the diagnosis of classical endometrial cancer. Contrary to the cases reported in the literature, the incidental diagnosis of EGCC in our patient was made based on an abnormal pap smear in an asymptomatic woman. This incidental finding probably allowed earlier diagnosis of the neoplasm, which correlated with a non-metastatic tumor, and therefore, with a better prognostic outcome. There is no clear consensus for the management of EGCC, with hysterectomy often being the treatment of choice, occasionally associated with other treatments, such as radiation. As described in Table 1, 11 patients had stage I disease, 3 had stage III, and 1 presented with stage IV. The treatment was radiation followed by hysterectomy in 3 cases; radiation alone in 2 cases; hysterectomy and salpingo-oophorectomy alone in 1 case, in 1 case followed by radiation, in 6 cases associated with pelvic node dissection, of which 2 cases were followed by radiotherapy; 2 cases were treated with hormonal therapy, 1 alone and 1 in association with surgery and radiation. In

Table 1. Characteristics of patients with endometrial glassy cell carcinoma, therapy and follow up, as reported in the literature

\begin{tabular}{|c|c|c|c|c|c|}
\hline Author & Age & Stage & Symptoms & Treatment & Follow-up \\
\hline \multirow[t]{5}{*}{ Christopherson et al. [6] } & 56 & । & Vaginal bleeding & $\mathrm{RT}+\mathrm{H}$ & DOD at 5 months \\
\hline & 70 & । & Vaginal bleeding & $\mathrm{RT}+\mathrm{H}$ & DOD at 32 months \\
\hline & 71 & । & Vaginal bleeding & $\mathrm{RT}+\mathrm{H}$ & Death of pneumonia after 6 years-NED \\
\hline & 78 & । & Vaginal bleeding & RT & Suicide after 7 months-no info on disease \\
\hline & 78 & III & Vaginal bleeding & RT & DOD after 5 months \\
\hline Arends et al. [7] & 59 & IIIA & Vaginal bleeding & TAHBSO+RT+Progestins & NED after 24 months \\
\hline Dawson et al. [8] & 96 & IVB & Vaginal bleeding & Megestrol & NED after 16 months \\
\hline \multirow[t]{3}{*}{ De Rosa et al. [9] } & 58 & $\mathrm{IB}$ & Metrorrhagia & TAHBSO & DOD after 12 months \\
\hline & 52 & $\mathrm{IB}$ & Metrorrhagia & TAHBSO+PND & DOD after 16 months \\
\hline & 58 & $\mathrm{IB}$ & Metrorrhagia & TAHBSO+PND & ED after 36 months \\
\hline Hachisuga et al. [10] & 62 & IIIC & Vaginal bleeding & TAHBSO+PND+RT & NED after 66 months \\
\hline Mhawech et al. [11] & 60 & $\mathrm{IB}$ & Vaginal bleeding & TAHBSO+PND+RT & NED after 60 months \\
\hline Ferrandina et al. [12] & 63 & $\mathrm{IB}$ & Vaginal bleeding & TAHBSO+PND & NED after 69 months \\
\hline Nagy and Sipos [13] & 68 & IA & & TAHBSO+RT & NED after 24 months \\
\hline Current case & 64 & $\mathrm{IA}$ & Abnormal pap smear & TAHBSO+PND & NED after 14 months \\
\hline
\end{tabular}

$\mathrm{RT}$, radiotherapy; $\mathrm{H}$, hysterectomy; TAHBSO, total abdominal hysterectomy bilateral salpingo-oophorectomy; PND, pelvic node dissection; DOD, death of disease; ED, alive with evidence of disease; NED, no evidence of disease. 


\section{Obstetrics \& Gynecology Science}

2 cases $[7,8]$, hormonal therapy was the choice and the patients were treated successfully with hormonal therapy, suggesting the presence of, at least, progesterone receptors. In our case, similarly to those reported by Ferrandina et al. [12] and Mhawech et al. [11], estrogen and progesterone receptors were negative.

Few studies reported presence of hormonal receptors and performed immunohistochemical analysis. Ferrandina et al. [12] reported strong positivity for Ki67 and vascular endothelial growth factor, weak positivity for p53, Her2/Neu, and very weak positivity for bcl-2 protein, while estrogen and progesterone receptors were negative. Mhawech et al. [11] reported positivity for vimentin, neuron-specific enolase, protein gene product 9.5, p53, and Ki67, while chromogranin $A$ and $B, C E A$, smooth muscle actin, muscle-specific actin, CAM 5.2, 34bE12, Leu-5, CK7, CK19, CK20, CK22, desmoplakin, desmin, myoglobin, human epidermal growth factor receptor 2 protein, and estrogen and progesterone receptors were negative.

Despite the fact that cervical GCC is known to be associated with poor prognosis; currently, the specific prognosis of EGCC remains unknown. Particularly, it is not possible to arrive at any conclusion considering the limited data available and the small sample size of the reported studies [6-13].

Follow-up period in the reported studies ranged from 5 to 69 months. In case of patients at stage I at the moment of surgery, 4 patients died of the disease within 16 months, while 2 patients died due to other causes. In particular, one of these died after 6 years form surgery and no sign of endometrial cancer was detected at the autopsy. Four patients were alive with no evidence of the disease; and 1 patient was alive with the disease. Excluding one patient who committed suicide, 5 patients out of 11 (45.5\%) were disease free (at minimum of 14 months). Regarding stage III, 2 patients out of 3 were alive without the disease (at 24 and 66 months), and the only patient with stage IV was alive after 16 months.

In conclusion, there is limited data pertaining to EGCC, with no standard guidelines to aid in therapeutic decisionmaking and counseling of patients.

We have therefore described a case of EGCC considering that, publication of more cases would aid in defining more strict and shared diagnostic criteria and better understand the course, ideal treatment, and accurate prognosis of this disease.

\section{Conflict of interest}

No potential conflict of interest relevant to this article was reported.

\section{Ethical approval}

This article does not contain any studies conducted on animals performed by any of the authors.

We do not have a IRB number for approval: the patient consented to the use of her personal and medical data at the hospital admission. In addition, she signed a specific consent to authorize the publication of her case.

\section{Patient consent}

Informed consent was obtained from the patient included in this study and data were anonymized.

\section{REFERENCES}

1. Cherry CP, Glucksmann A. Incidence, histology, and response to radiation of mixed carcinomas (adenoacanthomas) of the uterine cervix. Cancer 1956;9:971-9.

2. Seltzer V, Sall S, Castadot MJ, Muradian-Davidian M, Sedlis A. Glassy cell cervical carcinoma. Gynecol Oncol 1979;8:141-51.

3. Tamimi HK, Ek M, Hesla J, Cain JM, Figge DC, Greer BE. Glassy cell carcinoma of the cervix redefined. Obstet Gynecol 1988;71:837-41.

4. Aru A, Rasmussen LA, Federspiel B, Horn T. Glassy cell carcinoma of the colon with human chorionic gonadotropin-production. A case report with immunohistochemical and ultrastructural analysis. Am J Surg Pathol 1996;20:187-92.

5. Herbold DR, Axelrod JH, Bobowski SJ, Freel JH. Glassy cell carcinoma of the fallopian tube. A case report. Int J Gynecol Pathol 1988;7:384-90.

6. Christopherson WM, Alberhasky RC, Connelly PJ. Glassy cell carcinoma of the endometrium. Hum Pathol 1982;13:418-21.

7. Arends JW, Willebrand D, DeKoning Gans HJ, Swaen GJ, 


\section{Obstetrics \& Gynecology Science}

Vol. 62, No. 6, 2019

Bosman FT. Adenocarcinoma of the endometrium with glassy-cell features-immunohistochemical observations. Histopathology 1984;8:873-9.

8. Dawson EC, Belinson JL, Lee K. Glassy cell carcinoma of the endometrium responsive to megestrol acetate. Gynecol Oncol 1989;33:121-4.

9. De Rosa G, Barra E, Palombini L, Nappi C. Glassy cell carcinoma of the endometrium. Pathologica 1987;79:33945.

10. Hachisuga T, Sugimori H, Kaku T, Matsukuma K, Tsukamoto N, Nakano H. Glassy cell carcinoma of the endometrium. Gynecol Oncol 1990;36:134-8.

11. Mhawech P, Dellas A, Terracciano LM. Glassy cell carcinoma of the endometrium: a case report and review of the literature. Arch Pathol Lab Med 2001;125:816-9.
12. Ferrandina G, Zannoni GF, Petrillo M, Vellone V, Martinelli E, Scambia G. Glassy cell carcinoma of the endometrium: a case report and review of the literature. Pathol Res Pract 2007;203:217-20.

13. Nagy PS, Sipos J. Glassy cell carcinoma of the endometrium: case report and review of the literature. Orv Hetil 2009;150:1173-6.

14. Kurman RJ, Hedrick Ellenson L, Ronnett BM. Blaunstein's pathology of the female genital tract. 6th ed. New York (NY): Springer Nature; 2011.

15. Nordström B, Strang P, Bergström R, Nilsson S, Tribukait B. A comparison of proliferation markers and their prognostic value for women with endometrial carcinoma. Ki67, proliferating cell nuclear antigen, and flow cytometric S-phase fraction. Cancer 1996;78:1942-51. 